\title{
Incidence of SARS-CoV-2 Infection among COVID-19 Vaccinated and Unvaccinated Healthcare Personnel, First Responders, and other Essential and Frontline Workers - Eight U.S. Locations, January-September 2021
}

\author{
Allison Naleway ${ }^{1}$, Lauren Grant ${ }^{2}$, Alberto Caban-Martinez ${ }^{3}$, Meredith Wesley ${ }^{4}$, Jefferey \\ Burgess $^{5}$, Kimberly Groover ${ }^{6}$, Manjusha Gaglani ${ }^{7}$, Sarang Yoon ${ }^{8}$, Harmony Tyner ${ }^{9}$, \\ Jennifer Meece ${ }^{10}$, Jennifer Kuntz ${ }^{1}$, Young Yoo ${ }^{2}$, Natasha Schaefer-Solle ${ }^{3}$, Lauren Olsho ${ }^{11}$, \\ Joe Gerald ${ }^{12}$, Spencer Rose ${ }^{13}$, Matthew Thiese ${ }^{8}$, Jessica Lundgren ${ }^{9}$, Holly Groom ${ }^{1}$, \\ Josephine Mak ${ }^{2}$, Paola Feliciano ${ }^{3}$, Laura Edwards ${ }^{6}$, Karen Lutrick ${ }^{12}$, Kayan Dunnigan ${ }^{13}$, \\ Andrew Phillips ${ }^{8}$, Marilyn Odean ${ }^{14}$, Katherine Ellingson ${ }^{12}$, Karley Respet ${ }^{9}$, Mark \\ Thompson $^{2}$, and Ashley Fowlkes ${ }^{2}$ \\ ${ }^{1}$ Kaiser Permanente Center for Health Research Northwest Region \\ ${ }^{2}$ Centers for Disease Control and Prevention \\ ${ }^{3}$ University of Miami Miller School of Medicine \\ ${ }^{4} \mathrm{CDC}$ \\ ${ }^{5}$ University of Arizona Medical Center - University Campus \\ ${ }^{6}$ ABT Associates Inc \\ ${ }^{7}$ Baylor Scott \& White Health, Texas A\&M HSC COM \\ ${ }^{8}$ University of Utah Health \\ ${ }^{9}$ St. Luke's Regional Health Care System \\ ${ }^{10}$ Marshfield Clinic Research Institute \\ ${ }^{11}$ Abt Associates, Inc. \\ ${ }^{12}$ The University of Arizona Mel and Enid Zuckerman College of Public Health \\ ${ }^{13}$ Baylor Scott \& White Health \\ ${ }^{14}$ Whiteside Institute for Clinical Research, St. Luke's
}

December 2, 2021

\begin{abstract}
Background. We sought to evaluate the impact of changes in estimates of COVID-19 vaccine effectiveness on the incidence of laboratory-confirmed infection among frontline workers at high risk for SARS-CoV-2. Methods. We analyzed data from a prospective frontline worker cohort to estimate the incidence of COVID-19 by month as well as the association of COVID-19 vaccination, occupation, demographics, physical distancing and mask use with infection risk. Participants completed baseline and quarterly surveys, and each week self-collected mid-turbinate nasal swabs and reported symptoms. Results. Among 1,018 unvaccinated and 3,531 fully vaccinated workers, the monthly incidence of laboratory-confirmed SARS-CoV-2 infection in January 2021 was 13.9 (95\% confidence interval [CI]: 10.4-17.4), declining to 0.5 (95\% CI -0.4-1.4) per 1000 person-weeks in June. By September 2021, when the Delta variant predominated, incidence had once again risen to 13.6 (95\% CI 7.8-19.4) per 1000 person-weeks. In contrast, there was no reportable incidence among fully vaccinated participants at the end of January
\end{abstract}


2021, and incidence remained low until September 2021 when it rose modestly to 4.1 (95\% CI 1.9-3.8) per 1000. Below average facemask use was associated with a higher risk of infection for unvaccinated participants during exposure to persons who may have COVID-19, and vaccinated participants during hours in the community. Conclusions. COVID-19 vaccination was significantly associated with a lower risk of SARS-CoV-2 infection despite Delta variant predominance. Our data demonstrate the added protective benefit of facemask use among both unvaccinated and vaccinated frontline workers.

\section{Hosted file}

Naleway_Incidence_VaccineEra_IORV_112421.docx available at https://authorea.com/users/ 449050/articles/547695-incidence-of-sars-cov-2-infection-among-covid-19-vaccinated-andunvaccinated-healthcare-personnel-first-responders-and-other-essential-and-frontlineworkers-eight-u-s-locations-january-september-2021

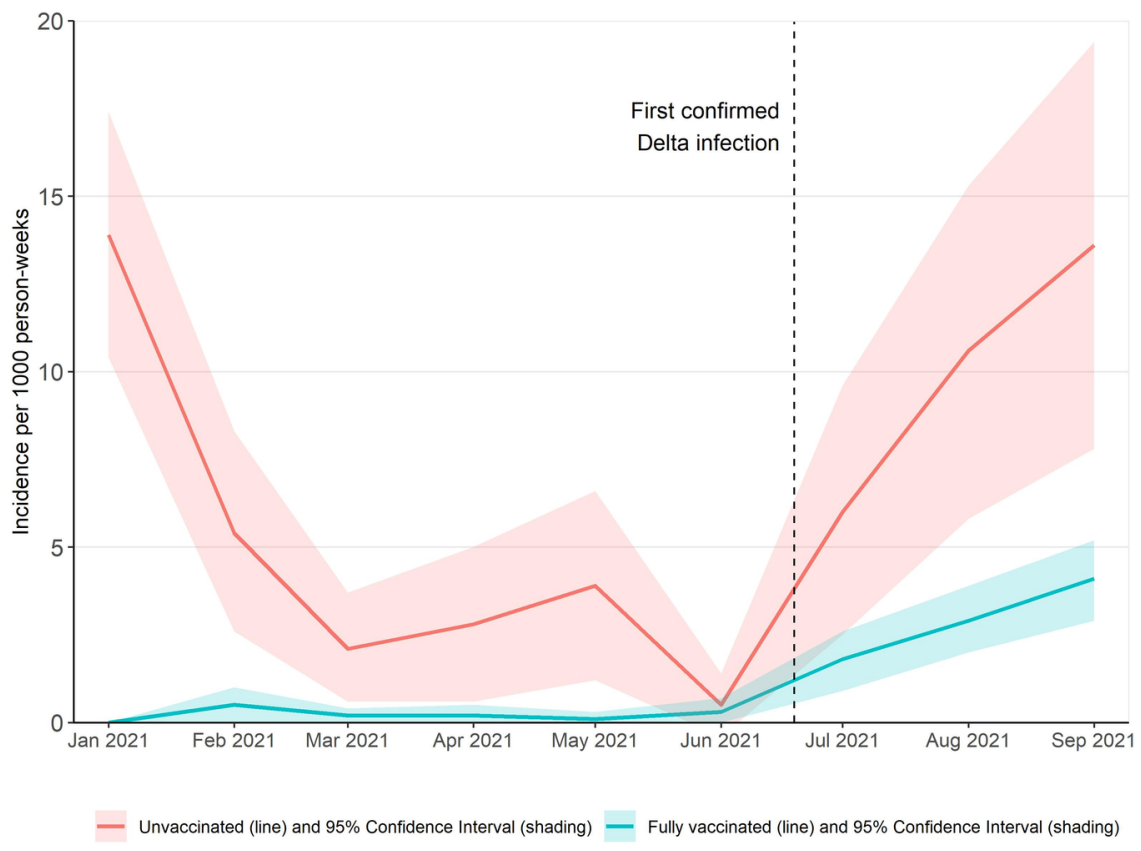

